\title{
Gastrointestinal Bioaccessibility and Colonic Fermentation of Fucoxanthin from the Extract of the Microalga Nitzschia laevis
}

\author{
Bingbing Guo, ${ }^{\dagger, \ddagger}$ Teresa Oliviero, ${ }^{\ddagger}$ Vincenzo Fogliano, ${ }^{\ddagger}$ Y Yuwei Ma, ${ }^{\ddagger}$ Feng Chen, ${ }^{*}, \dagger, \S$ \\ and Edoardo Capuano*, \\ †Institute for Food and Bioresource Engineering, College of Engineering, Peking University, Beijing 100871, People’s Republic of
China
${ }^{\ddagger}$ Food Quality and Design Group, Department of Agrotechnology and Food Sciences, Wageningen University, Bornse Weilanden 9,
6708 WG Wageningen, Netherlands
${ }^{\S}$ Institute for Advanced Study, Shenzhen University, Nanshan District, Shenzhen, Guangdong 518060, People’s Republic of China
}

Supporting Information

ABSTRACT: The extract of microalga Nitzschia laevis (NLE) is considered a source of dietary fucoxanthin, a carotenoid possessing a variety of health benefits. In the present study, the bioaccessibility and deacetylation of fucoxanthin were studied by simulated in vitro gastrointestinal digestion and colonic batch fermentation. In the gastric phase, higher fucoxanthin loss was observed at $\mathrm{pH} 3$ compared to $\mathrm{pH} 4$ and 5. Lipases are crucial for the deacetylation of fucoxanthin into fucoxanthinol. Fucoxanthinol production decreased significantly in the order: pure fucoxanthin $(25.3 \%)>$ NLE $(21.3 \%)>$ fucoxanthincontaining emulsion (11.74\%). More than $32.7 \%$ of fucoxanthin and fucoxanthinol was bioaccessible after gastrointestinal digestion of NLE. During colon fermentation of NLE, a higher loss of fucoxanthin and changes of short-chain fatty acid production were observed but no fucoxanthinol was detected. Altogether, we provided novel insights on the fucoxanthin fate along the human digestion tract and showed the potential of NLE as a promising source of fucoxanthin.

KEYWORDS: fucoxanthin, microalgae, digestion, fermentation

\section{INTRODUCTION}

Fucoxanthin, accounting for $10 \%$ of total carotenoids in nature, ${ }^{1}$ is an algal carotenoid. It possesses a variety of healthbeneficial bioactivities: antioxidant, antidiabetes, anticarcinogenic, antiallergic, anti-inflammatory, and antiosteoporotic activities, among others. ${ }^{2}$ Particularly, both mice and human studies have proven fucoxanthin effective in reducing body weight. $^{3-5}$ Therefore, fucoxanthin-containing products are now widely available on the market as functional food supplements, predominantly indicated for weight loss.

Fucoxanthin is mainly found in brown algae (seaweeds) and diatoms (microalgae). Most fucoxanthin-containing products that are available are brown algae extracts supplemented with oil and/or polyphenols from tea or other sources. However, the fucoxanthin content in brown algae is much lower than that of microalgae. ${ }^{6}$ Moreover, environmental issues, such as land occupation, extraction technology, byproduct waste, and contamination, are to be considered when dealing with production of fucoxanthin from brown algae. ${ }^{7-9}$ Fucoxanthin can be effectively produced by microalgae: Fucovital, a patented, all-natural 3\% fucoxanthin oleoresin produced and extracted from Phaeodactylum tricornutum, was granted New Dietary Ingredient Notification (NDIN, 1048, 2017) by the U.S. Food and Drug Administration (FDA). Microalgae, diatoms, specifically, have also been screened to produce fucoxanthin. ${ }^{6}$ Among them, notably, Nitzschia laevis is a very productive microalga, which was also successfully cultured heterotrophically on glucose. ${ }^{10}$ The biomass concentration of $N$. laevis in the fermenter could reach $17.25 \mathrm{~g} / \mathrm{L}$, and the fucoxanthin productivity of mixed-cultured N. laevis could be as high as $16.5 \mathrm{mg} \mathrm{L}^{-1}$ day $^{-1}$, which is the highest reported for diatoms up to now. ${ }^{11}$ Moreover, other functional ingredients, including carotenoids (e.g., diadinoxanthin, diatoxanthin, $\beta$ carotene, and lycopene), polyphenols, and lipids [especially polyunsaturated fatty acids (PUFAs)], are usually present in the extract of $N$. laevis. ${ }^{12}$ All of these make this extract a potential sustainable source of fucoxanthin.

Bioavailability studies reported that most fucoxanthin is absorbed as fucoxanthinol. ${ }^{13}$ A study on mice reported that fucoxanthin was converted to fucoxanthinol in the gastrointestinal tract through deacetylation, possibly by lipase or cholesterol esterase, ${ }^{13}$ and fucoxanthinol was then converted to amarouciaxanthin by de-epoxidation in the liver in mice. ${ }^{14}$ Fucoxanthinol was detected in the liver, the lung, the spleen, and the heart, while amarouciaxanthin was accumulated in the adipose tissue. ${ }^{14}$ Human studies have been conducted with fucoxanthin from brown algae, and both fucoxanthin and fucoxanthinol have been detected in plasma. ${ }^{15}$ However, very little is known about the behavior of fucoxanthin in the human digestion tract and the factors affecting its bioaccessibility and conversion into fucoxanthinol from fucoxanthin-containing products. In particular, fucoxanthin is easily oxidized in the

Special Issue: Food Bioactives and Health

Received: April 21, 2019

Revised: May 11, 2019

Accepted: May 13, 2019

Published: May 13, 2019 
presence of oxygen and is unstable in acidic conditions, ${ }^{16}$ indicating a possibility that fucoxanthin could be lost during the gastric or small intestinal digestion. Also, different bioaccessibilities of fucoxanthin were observed for fucoxanthin-containing capsules and milk powder supplemented with different oils. ${ }^{17,18}$ Therefore, the inherent stability of fucoxanthin and the interaction with other compounds (e.g., lipids) during digestion needs to be considered.

It is widely recognized that a substantial, if not predominant, part of dietary carotenoids escapes absorption in the small intestine and ends up in the colon, where they can shape gut microbiota ${ }^{19}$ or gut metagenome. ${ }^{20}$ Specifically, the ability of the fucoxanthin-containing extract of $N$. laevis to change the gut microbiota composition when administered to mice for 2 months was reported. ${ }^{12}$ In addition, gut microbiota affected carotenoid bioavailability by altering the carotenoid absorption or degradation patterns. ${ }^{21}$ A previous study reported that gut microbiota may contribute to $11 \%$ of variance to the serum xanthophyll concentration. ${ }^{22}$ However, how fucoxanthin escaping absorption in the small intestine would interact with gut microbiota, e.g., whether it is metabolized or not, needs to be clarified.

In the present study, fucoxanthin deacetylation and its bioaccessibility from the extract of $N$. laevis were investigated using simulated in vitro digestion models. The effect of gastric $\mathrm{pH}$, kinetics of deacetylation, and bioaccessibility in the small intestine were investigated. Moreover, fucoxanthin recovery and the effect on short-chain fatty acid (SCFA) production were, for the first time, analyzed through in vitro colonic fermentation with human feces.

\section{MATERIALS AND METHODS}

Chemicals and Reagents. Pure fucoxanthin (PF, 16337), porcine pepsin (P6887), porcine pancreatin (P1750, 4× USP), porcine bile salt preparation (B8631), orlistat (04139), and butylated hydroxytoluene (BHT) were purchased from Sigma-Aldrich (Merck $\mathrm{KGaA}$, Germany). Olive oil (extra virgin, Carapelli, Dallas, TX, U.S.A.) was bought from a local market in the Netherlands. KCl, $\mathrm{KH}_{2} \mathrm{PO}_{4}, \mathrm{NaCl}, \mathrm{MgCl}_{2} \cdot\left(\mathrm{H}_{2} \mathrm{O}\right)_{6}$, and $\mathrm{CaCl}_{2} \cdot\left(\mathrm{H}_{2} \mathrm{O}\right)_{2}$, and pure ethanol were purchased from VWR International B.V. (Netherlands). $\mathrm{KH}_{2} \mathrm{PO}_{4}, \mathrm{NaCl},\left(\mathrm{NH}_{4}\right)_{2} \mathrm{CO}_{3}, \mathrm{NaOH}, \mathrm{HCl}$, and Tween 80 were purchased from Sigma-Aldrich Chemie B.V. (Netherlands) as well as the yeast extract, peptone, mucine, and L-cysteine $\mathrm{HCl}$. Acetonitrile and chloroform were bought from LPS B.V. (Oss, Netherlands). All of the chemicals used are of analytical and chromatographic grade.

Preparation of $\mathbf{N}$. laevis Extract (NLE). The NLE was obtained according to the method described in our previous paper. ${ }^{6}$ Briefly, about $100 \mathrm{mg}$ of lyophilized powder was ground with liquid nitrogen. Immediately, $10 \mathrm{~mL}$ of ethanol was added to the ground cells for extraction, followed by end-to-end shaking for $1 \mathrm{~h}$. Supernatants were collected by centrifugation $\left(2500 \mathrm{~g}\right.$ for $5 \mathrm{~min}$ at $\left.4{ }^{\circ} \mathrm{C}\right)$. The extraction was conducted twice, and the supernatants were combined. NLE was obtained by evaporating with nitrogen at $22^{\circ} \mathrm{C}$. All of the procedures were carried out under red light. The composition of NLE was investigated in our previous study. ${ }^{12}$ The fucoxanthin content was about $5.1 \%$, and no fucoxanthinol is detected in the freshly extracted NLE. Notably, there are more than $7 \%$ lipids in NLE.

Preparation of the Fucoxanthin-Containing Emulsion (FCE). The FCE was prepared as previously described ${ }^{23}$ by mixing (13 500 rpm for 10 min, UltraTurrax, IKA-Werke, Staufen, Germany) pure fucoxanthin and olive oil $(5 \%, \mathrm{w} / \mathrm{w})$ with an aqueous solution containing Tween $80(1 \%, \mathrm{w} / \mathrm{w})$. The obtained FCE was stored at $-20{ }^{\circ} \mathrm{C}$ in the dark and used within 3 days.

Simulated In Vitro Gastrointestinal Digestion. All samples were digested using a static in vitro digestion system consisting of a simulated oral phase, gastric phase, and intestinal phase, with modifications. $^{24,25}$ The compositions (\%, w/w) of the simulated salivary fluid (SSF), simulated gastric fluid (SGF, pH $3.0 \pm 0.05$ ), and simulated intestinal fluid (SIF, pH $7.0 \pm 0.05$ ) were as reported. ${ }^{24}$

For the oral phase, for $5 \mathrm{~mL}$ samples (fucoxanthin content of 100 $\mu \mathrm{g}), 3.5 \mathrm{~mL}$ of SSF stock solution $\left(37^{\circ} \mathrm{C}\right), 25 \mu \mathrm{L}$ of $\mathrm{CaCl}_{2}(0.3 \mathrm{M})$, and $1.475 \mathrm{~mL}$ of Milli-Q water (Veolia water, Veolia Water Solutions and Technologies Netherlands B.V.) were added and mixed. For the experiment of oxidation prevention, 0.02 and $0.04 \%$ BHT were added.

Subsequently, to start the gastric phase, $5 \mathrm{~mL}$ of oral bolus was mixed with $3.75 \mathrm{~mL}$ of SGF $\left(37^{\circ} \mathrm{C}\right)$. Then, $0.8 \mathrm{~mL}$ of porcine pepsin stock solutions of 25000 units $/ \mathrm{mL}$ ( 2000 units $/ \mathrm{mL}$ in final chyme) in SGF $\left(37^{\circ} \mathrm{C}\right)$ was added, followed by $2.5 \mu \mathrm{L}$ of $\mathrm{CaCl}_{2}(0.3 \mathrm{M})$. Last, about $0.1 \mathrm{~mL}$ of $\mathrm{HCl}(1 \mathrm{M})$ was added to reach $\mathrm{pH} 3$. Finally, 0.349 $\mathrm{mL}$ of Milli- $\mathrm{Q}$ water was added to obtain the final volume $(10 \mathrm{~mL})$. Then, the chyme was shaken at $37{ }^{\circ} \mathrm{C}$ for $2 \mathrm{~h}$ without light. All reactors were filled with nitrogen when closed before starting the digestion. In the experiments at different gastric $\mathrm{pH}, \mathrm{pH}$ was adjusted to 4 and 5 before starting the gastric phase with $\mathrm{HCl}$.

At the end of the gastric digestion step, about $0.075 \mathrm{~mL}$ of $\mathrm{NaOH}$ ( $1 \mathrm{M})$ was added to reach $\mathrm{pH} 7.0$, inhibiting the gastric enzyme activity. Thereafter, to $10 \mathrm{~mL}$ of gastric chyme, $5.5 \mathrm{~mL}$ of SIF (37 ${ }^{\circ} \mathrm{C}$ ), $2.5 \mathrm{~mL}$ of porcine pancreatin (trypsin activity of 800 units $/ \mathrm{mL}$ and lipase activity of around 280 units $/ \mathrm{mg})$ in $\operatorname{SIF}\left(37^{\circ} \mathrm{C}\right), 1.25 \mathrm{~mL}$ of fresh bile stock solutions ( $10 \mathrm{mM}$ in the final intestinal chyme), 20 $\mu \mathrm{L}$ of $\mathrm{CaCl}_{2}(0.3 \mathrm{M})$, and $0.655 \mathrm{~mL}$ of Milli-Q water were added. Finally, the chyme was incubated in a shaking water bath for $2 \mathrm{~h}$ at 37 ${ }^{\circ} \mathrm{C}$, and the digestion was stopped by cooling immediately in an ice bath. To study the kinetics of fucoxanthin micellarization, samples were collected at $0,10,20,30,60$, and $120 \mathrm{~min}$, respectively. For the inhibition of lipase activity, $10 \mu \mathrm{g} / \mathrm{mL}$ orlistat was added to pancreatin dissolved in SSF and the mixture was incubated at $37{ }^{\circ} \mathrm{C}$ for $30 \mathrm{~min}$ before undertaking the simulated pancreatic digestion. ${ }^{26}$ In this experiment, samples were only collected after $120 \mathrm{~min}$. Also, for the experiment with BHT, samples were collected at the end of intestinal digestion. All intestinal digests were centrifuged for $10 \mathrm{~min}$ at $20000 \mathrm{~g}$ $\left(4{ }^{\circ} \mathrm{C}\right)$, and the supernatants were collected and filtered through a $0.22 \mu \mathrm{m}$ membrane filter (Phenomenex, Netherlands). Fucoxanthin in the filtered supernatants was supposed to have been incorporated into the micellar phase. All tubes used here were filled with nitrogen before starting the digestion procedure, and all procedures were conducted under red light.

In Vitro Colonic Fermentation. Fresh fecal samples were donated by three healthy adults [two Chinese and one Dutch, aged 23-28 years old, with a body mass index (BMI) of 18.5-23.9], who declared no smoking and no antibiotic consumption for 6 months before the beginning of the study. The fecal samples were prepared according to protocols previously reported, with modifications. ${ }^{27,28}$ Briefly, about $20.0 \mathrm{~g}$ of feces was diluted by $100 \mathrm{~mL}$ of anaerobic phosphate buffer before homogenization in a stomacher bag. The resulting fecal suspension was filtered and considered as the fecal microbiota inocula. The basal medium, consisting of $5.22 \mathrm{~g} / \mathrm{L}$ $\mathrm{K}_{2} \mathrm{HPO}_{4}, 16.32 \mathrm{~g} / \mathrm{L} \mathrm{KH} \mathrm{PO}_{4}, 2.0 \mathrm{~g} / \mathrm{L} \mathrm{NaHCO} 3,2.0 \mathrm{~g} / \mathrm{L}$ yeast extract, $2.0 \mathrm{~g} / \mathrm{L}$ peptone, $1.0 \mathrm{~g} / \mathrm{L}$ mucine, $0.5 \mathrm{~g} / \mathrm{L}$ L-cysteine $\mathrm{HCl}$, and 2.0 $\mathrm{mL} / \mathrm{L}$ tween 80 , was used after 30 min flushed with nitrogen before autoclaving. Fucoxanthin $(100 \mu \mathrm{g})$ from all fucoxanthin-containing products dissolved in SIF was added, together with a $10 \%$ inoculation. The fermentation was then started at $37^{\circ} \mathrm{C}$ with continuous shaking (60 rpm) under anaerobic conditions without light. In parallel, two different controls were conducted under the applied conditions: (i) the fucoxanthin-containing fractions were fermented without inoculation to determine possible chemical reactions, and (ii) the fecal suspension was fermented without fucoxanthin-containing products to be served as a negative control. All incubations were performed in triplicate and sampled at $0,2,10,24,48$, and $72 \mathrm{~h}$. All of the samples were centrifuged immediately when sampled, and the supernatants were stored at $-20{ }^{\circ} \mathrm{C}$ until use.

Fucoxanthin Analysis. For the in vitro gastrointestinal digestion samples, fucoxanthin and its metabolites were extracted as previously described, ${ }^{17}$ with tiny modifications. A total of $10 \mathrm{~mL}$ of digest was mixed with $5 \mathrm{~mL}$ of chloroform as well as $0.5 \mathrm{~g}$ of $\mathrm{NaCl}$, followed by 

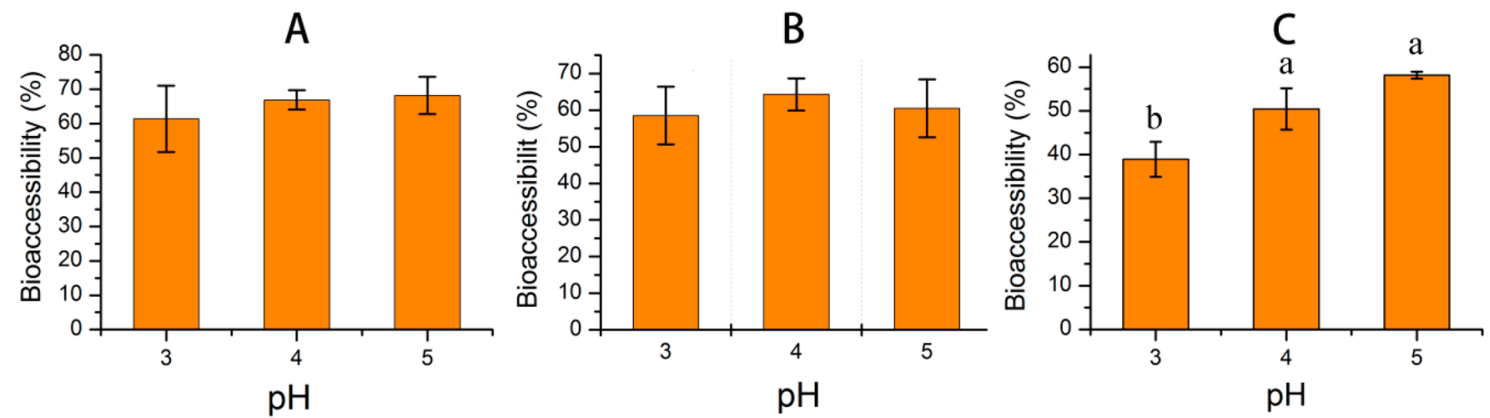

Figure 1. Effect of gastric $\mathrm{pH}$ on the recovery of fucoxanthin: (A) PF, (B) FCE, and (C) NLE. All of the data were from three replications and expressed as the mean \pm SD. In each panel, different lowercase letters indicate statistical differences in recovery at $p<0.05$.

end-to-end shaking for $30 \mathrm{~min}$. Then, the chloroform layer was collected through centrifugation (4700 rpm for $15 \mathrm{~min}$ at $4{ }^{\circ} \mathrm{C}$ ). The extraction was repeated twice, and both of the chloroform fractions were combined. Chloroform was then evaporated with nitrogen, and the samples were redissolved in ethanol before analysis with highperformance liquid chromatography (HPLC). The same method was applied to the supernatants in the batch fermentation experiments (see the previous section).

Fucoxanthin was determined and quantified using a HPLC-diode array detector (DAD) system (Ultimate 3000, Thermo Scientific, Netherlands) equipped with an Onyx monolithic C18 column $(100 \times$ $4.6 \mathrm{~mm}$, with a pore size of $130 \AA$, Phenomenex, Netherlands). The column temperature was kept at $30{ }^{\circ} \mathrm{C}$. The mobile phase was a mixture of acetonitrile and water (85:15), and the flow rate was 1.5 $\mathrm{mL} / \mathrm{min}$. The injection volume was $10 \mu \mathrm{L}$. Fucoxanthin and fucoxanthinol were identified at $450 \mathrm{~nm}$ and quantified using external calibration curves.

The bioaccessibility/recovery of fucoxanthin was defined as the sum of fucoxanthin and fucoxanthinol contents that are detected in the supernatants, divided by total fucoxanthin initially present in the samples before digestion. Specific calculation was as follows:

$$
\begin{aligned}
& \text { bioaccessibility/recovery (\%) } \\
& =\frac{C_{\mathrm{m}, \text { fucoxanthin }}+C_{\mathrm{m} \text {,fucoxanthinol }}}{C_{\text {fucoxanthin }}} \times 100
\end{aligned}
$$

where $C_{\mathrm{m}}$ represents the fucoxanthin or fucoxanthinol amount in the micellar phase and $C_{\text {fucoxanthin }}$ represents the total fucoxanthin content $(\mu \mathrm{g})$ in the samples.

SCFA Analysis. Fecal-fermented samples $(1 \mathrm{~mL})$ were thawed and centrifuged at $9000 \mathrm{~g}$ for $5 \mathrm{~min}$ at $4{ }^{\circ} \mathrm{C}$. The resulting supernatants were then filtered using a $0.2 \mu \mathrm{m}$ RC filter. A total of $250 \mu \mathrm{L}$ of internal standard $(0.45 \mathrm{mg} / \mathrm{mL}$ 2-ethylbutyic acid in $0.3 \mathrm{M} \mathrm{HC}$ and $0.9 \mathrm{M}$ oxalic acid) was added to $500 \mu \mathrm{L}$ of sample. Finally, the solutions were mixed and centrifuged at $20000 \mathrm{~g}$ for $4 \mathrm{~min}$ before injection to gas chromatography (GC-2014, Shimadzu, Hertogenbosch, Netherlands), equipped with a capillary fatty-acid-free Stabilwax-DA column $(1 \mu \mathrm{m} \times 0.33 \mathrm{~mm} \times 30 \mathrm{~m}$, Restek, Bellefonte, PA, U.S.A.), a flame ionization detector, and a split injector. The injection volume was $1.0 \mu \mathrm{L}$. The carrier gas was nitrogen, and the temperatures of the injector and detector were 100 and $250{ }^{\circ} \mathrm{C}$, respectively. The temperature profile starts with $100{ }^{\circ} \mathrm{C}$, then increased at $10.8{ }^{\circ} \mathrm{C} / \mathrm{min}$ to $180{ }^{\circ} \mathrm{C}$, and kept at $180{ }^{\circ} \mathrm{C}$ for $2 \mathrm{~min}$. Then, it increased at $50.00{ }^{\circ} \mathrm{C} / \mathrm{min}$ until $240{ }^{\circ} \mathrm{C}$ and kept at $240{ }^{\circ} \mathrm{C}$ for $2 \mathrm{~min}$. Standard solutions of acetic, propionic, butyric, valeric, isovaleric, and isobutyric acids in concentrations of $0.01-0.45 \mathrm{mg} / \mathrm{mL}$ were prepared and used for identification and quantification.

Statistical Analysis. All of the data was expressed as the mean \pm standard deviation (SD). A Shapiro-Wilks test was used for the evaluation of normality of distribution, and a Leven test was applied for the equality of variance. One-way analysis of variance (ANOVA) was performed using SPSS followed with Tukey test to evaluate differences between groups. All figures were drawn with Origin 8.5 (OriginLab Corporation, Northampton, MA, U.S.A.). Statistical significance was set at $p \leq 0.05$. All statistical analyses were performed with SPSS 23.0 (SPSS, Chicago, IL, U.S.A.).

\section{RESULTS AND DISCUSSION}

Effect of Gastric pH on the Recovery of Fucoxanthin. The effect of gastric $\mathrm{pH}$ on fucoxanthin recovery was tested, and the results were shown in Figure 1. No significant difference for fucoxanthin bioaccessibility was observed when $\mathrm{PF}$ and FCE were digested at $\mathrm{pH} 4$ and 5 compared to $\mathrm{pH} 3$. However, gastric bioaccessibility of fucoxanthin from NLE was significantly lower at $\mathrm{pH} 3$ compared to that of $\mathrm{pH} 4$ and 5 . This last result agrees with previous results showing that fucoxanthin exhibits the lowest stability at $\mathrm{pH}<3$, the highest

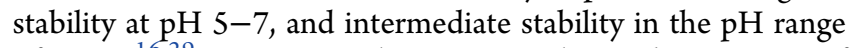
of $3-5 .^{16,29}$ Interestingly, a very limited amount of fucoxanthinol was detected in all samples after gastric digestion, indicating that the acidic environment of the stomach contributes very little to the deacetylation of fucoxanthin. However, because the fasted gastric $\mathrm{pH}$ is typically around 2 and can increase up to 5 or above in the fed state, ${ }^{30,31}$ consuming fucoxanthin after a meal might preserve a higher fraction of fucoxanthin because of the higher gastric $\mathrm{pH}$.

Data in Figure 1 also showed that the gastric recovery of fucoxanthin was about $40-70 \%$, which means that more than $30 \%$ fucoxanthin was not detected. Carotenoid degradation in acidic conditions is initiated by a largely unknown mechanism. ${ }^{16}$ Some scholars have argued that carotenoids may be degraded through protonation of the conjugated system in acidic conditions. ${ }^{32}$ However, more complicated mechanisms may account for the observed loss of fucoxanthin at acidic $\mathrm{pH}$ because the allene and carboxyl groups were not detected after acidic treatment. ${ }^{33}$ Also, we found that the gastric recovery of fucoxanthin from NLE was much lower compared to that of $\mathrm{PF}$ and FCE groups at $\mathrm{pH}$ 3. In this regard, other compounds in NLE might affect the recovery of fucoxanthin in this study. Further investigation on fucoxanthin changes in acids is needed. However, the observation that fucoxanthin could be degraded during the gastric digestion is important when developing fucoxanthin-based products.

Fucoxanthin Was Deacetylated into Fucoxanthinol by Pancreatic Lipase. Previous studies suggest that fucoxanthin is converted into fucoxanthinol by deacetylation during digestion, ${ }^{34,35}$ but a systematic account of the physiological factors modulating this transformation is still missing. We hypothesized that pancreatic lipase may have a primary role on the deacetylation of fucoxanthin to fucoxanthinol because of its esterase activity. Also, pancreatic 
lipase is essential for lipid digestion and carotenoid bioaccessibility by sustaining the formation of mixed micelles, where carotenoids are incorporated. To test this hypothesis, we run an additional in vitro intestinal digestion using orlistat, a known inhibitor of pancreatic lipases, and a complete inhibition was expected. ${ }^{26,36}$ Also, a further digestion was carried out without pancreatin.

The results are shown in Figure 2. About 53\% of initial fucoxanthin was detected as fucoxanthinol after $2 \mathrm{~h}$ of

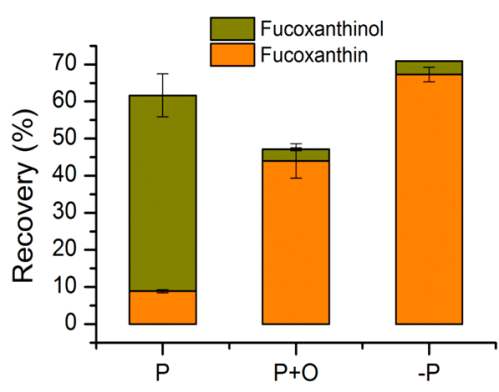

Figure 2. Effect of pancreatic lipase on the deacetylation of fucoxanthin during simulated in vitro intestinal digestion. Data are expressed as a percentage of the initial amount. All of the data were from three replications and expressed as the mean \pm SD. P, intestinal digestion with pancreatin; $\mathrm{P}+\mathrm{O}$, intestinal digestion with pancreatin and orlistat; and $-\mathrm{P}$, intestinal digestion without pancreatin.

digestion with pancreatin, whereas only about $3 \%$ of initial fucoxanthin was detected as fucoxanthinol after digestion with orlistat and after digestion without pancreatin. Thus, we concluded that pancreatic lipase mostly contributes to fucoxanthin deacetylation in this simulated gastrointestinal digestion. However, other digestive enzymes, such as carboxyl ester lipase, phospholipase A2, and pancreatic lipase-related proteins 1 and 2, possess esterase activity in the human small intestine too. Because inhibition of these enzymes by orlistat is also possible, ${ }^{37}$ we cannot exclude that they might also contribute to fucoxanthin deacetylation in vivo. ${ }^{38}$ Moreover, gastric lipase is produced in humans by chief cells of stomach mucosa that might also contribute to fucoxanthin deacetylation into fucoxanthinol in the gastric phase. This cannot be tested at the moment in an in vitro digestion model because of the lack of adequate replacers for human gastric lipase.

It is also worth noting that only $50-70 \%$ of initial fucoxanthin was recovered in mixed micelles after intestinal digestion, which is in line with previous reports that a partial incorporation of carotenoids in mixed micelles is observed. ${ }^{39,40}$ We hypothesized that part of the loss may been ascribed to oxidative degradation of fucoxanthin. Thus, a complete simulated digestion (including both gastric and intestinal phases) of PF was performed in the presence of 0.02 and $0.04 \%$ BHT (Figure 3); the addition of $0.04 \%$ BHT resulted in a $30 \%$ increase in the final fucoxanthin bioaccessibility. This confirms that part of the loss of fucoxanthin in the digestion model and possibly in vivo is due to oxidation.

Bioaccessibility of Fucoxanthin and Deacetylation Kinetics during Gastrointestinal Digestion. Bioaccessibility of fucoxanthin and deacetylation kinetics in different formulations were studied by performing a complete (gastric + intestinal) simulated digestion. Bioaccessibility values were 27.7, 27.5, and $32.7 \%$ for PF, FCE, and NLE, respectively (Figure 4). This is pretty consistent with the loss observed after individual gastric (Figure 1) and intestinal (Figure 2)

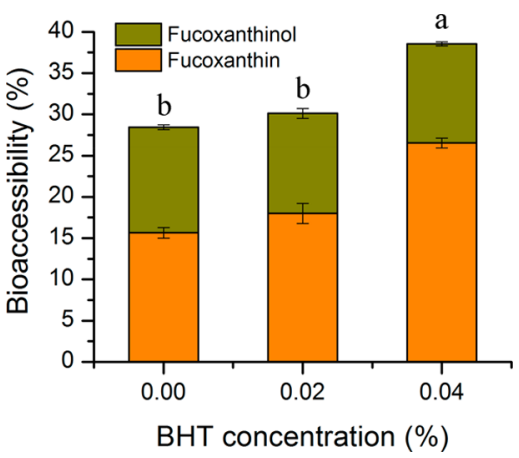

Figure 3. Effect of different concentrations of BHT on bioaccessibility of fucoxanthin during simulated in vitro intestinal digestion. All of the data were from three replications and expressed as the mean \pm SD. Different lowercase letters indicate statistical differences in bioaccessibility at $p<0.05$.

digestion. The kinetics of fucoxanthin deacetylation into fucoxanthinol for different samples is also illustrated in Figure 4. Overall, the total bioaccessibility (sum of fucoxanthin and fucoxanthinol recovery) and the deacetylation degree were clearly dependent upon the fucoxanthin source. At the beginning of the intestinal digestion, the total bioaccessibility is very high in FCE, intermediate in PF, and very low in NLE, indicating different gastric bioaccessibilities.

Specifically, in PF, fucoxanthinol was exponentially produced from fucoxanthin with concentrations (relative to the initial fucoxanthin content) increasing from 6.7 to $25.3 \%$ at the end of the intestinal digestion, while the fucoxanthin recovery decreased from 14.8 to $2.4 \%$ (Figure $4 \mathrm{~A}$ ), indicating that most fucoxanthin from PF samples was deacetylated into fucoxanthinol.

In FCE, the same exponential trend for fucoxanthin deacetylation was observed but less than half of fucoxanthin that entered into the intestinal phase was converted to fucoxanthinol, which is much less than that of the PF group (Figure 4B). Because pancreatic lipase is involved in both triglyceride hydrolysis and fucoxanthin deacetylation, ${ }^{25}$ we hypothesize that lipid digestion and fucoxanthin deacetylation might have competed with each other for lipase action. This might have resulted in the smaller transformation of fucoxanthin from FCE within the $60 \mathrm{~min}$. Also, fucoxanthin pre-emulsification in FCE did not increase the bioaccessibility of fucoxanthin compared to that of $\mathrm{PF}$, which seems to contradict studies indicating that emulsification assists carotenoid bioaccessibility, primarily by providing lipids for micellaration. ${ }^{41}$ However, lipids should be digested into free fatty acids and monoacylglycerides before being incorporated into mixed micelles. ${ }^{39}$ In this respect, the competition for lipase might also have resulted in less micellarization, which might have reduced the total bioaccessibility of fucoxanthin from FCE.

In NLE, within $20 \mathrm{~min}$, both fucoxanthin and fucoxanthinol contents in the intestinal supernatant increased. Afterward, fucoxanthinol was continuously produced, while fucoxanthin content began to fall down until the end of the intestinal phase (Figure 4C). The final fucoxanthin and fucoxanthinol bioaccessibilities were 11.4 and $21.3 \%$, respectively, indicating that about $2 / 3$ of fucoxanthin that entered into the small intestine was converted into fucoxanthinol, which is higher than that of FCE and lower than that of PF. Also, the total bioaccessibility of NLE fucoxanthin gradually but significantly 
A

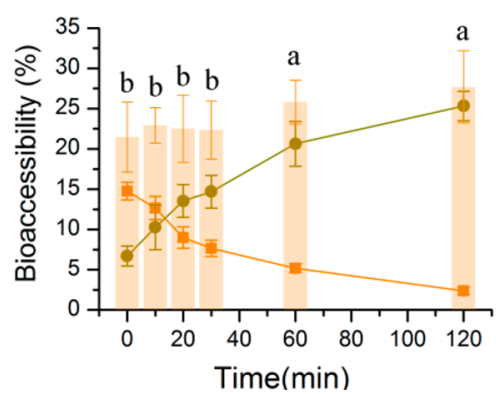

B

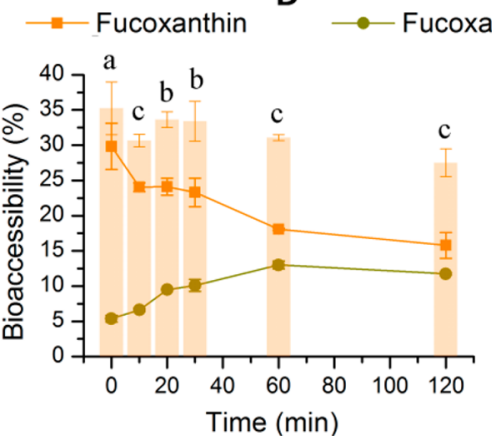

C

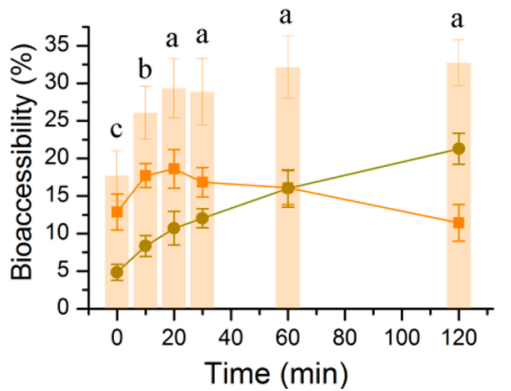

Figure 4. Bioaccessibility of fucoxanthin and deacetylation kinetics during simulated in vitro gastrointestinal digestion. The time course of fucoxanthin bioaccessibility is reported in the bar chart: (A) PF, (B) FCE, and (C) NLE. The column is the sum of fucoxanthin and fucoxanthinol contents. The lines indicated the fucoxanthin deacetylation: the orange line indicates the percentage of fucoxanthin, and the green line indicates the percentage of fucoxanthinol. All of the data were from three replications and expressed as the mean \pm SD. Different lowercase letters indicate a significant difference $(p<0.05)$ in fucoxanthin bioaccessibility.

increased during the intestinal digestion, and a higher bioaccessibility was obtained in comparison to that of PF and FCE at the end of intestinal digestion. In comparison to $\mathrm{PF}$ and FCE, NLE composition is more complex; i.e., other compounds are present that might have affected the deacetylation and promoted its bioaccessibility in the present study. However, specific mechanisms are unclear and need to be further studied.

Fucoxanthin Recovery during In Vitro Colonic Fermentation. NLE was added in the in vitro batch fermentation model with human feces, and the recovery of fucoxanthin was shown in Figure 5. A control was added,

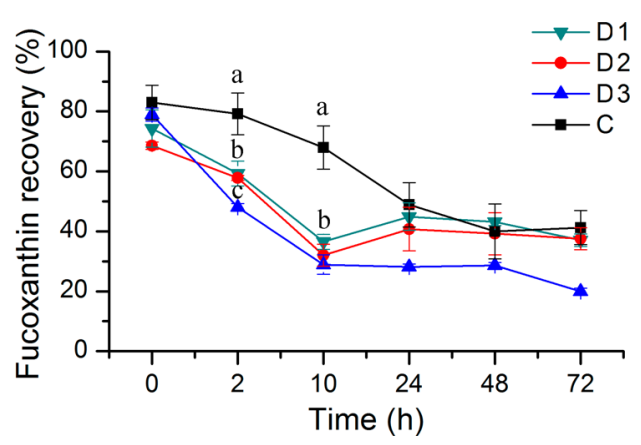

Figure 5. Fucoxanthin recoveries from the fermented supernatants during the in vitro colon fermentation. $\mathrm{C}$ represents fermentation of a basal medium with the addition of NLE but without microbiota inoculation. D1, D2, and D3 represent fermentation of the basal medium with the addition of NLE to microbiota of donors 1, 2, and 3, respectively. All of the data were from three replications and expressed as the mean $\pm \mathrm{SD}$. Different lowercase letters indicate statistical difference $(p<0.05)$ in the fucoxanthin content compared to the control at a specific time point.

where NLE was simply dissolved in the fermentation fluids without microbiota. Notably, loss of fucoxanthin from all treated groups and the control was observed. However, in comparison to the control, significant lower concentrations of fucoxanthin at times of $<10 \mathrm{~h}$ were observed when microbiota was present, suggesting that fucoxanthin might be metabolized/used by human microbiota. After $24 \mathrm{~h}$, fucoxanthin recovery from NLE reached a level comparable to that of the control group (Figure 5), which remained substantially unchanged until the end of the fermentation for microbiota from donors 1 and 2. With microbiota from donor 3, fucoxanthin recovery continued to decrease until the end of fermentation (Figure 5). Surprisingly, however, no fucoxanthinol was produced during the $72 \mathrm{~h}$ fermentation for all groups, indicating possibilities that either no fucoxanthin was deacetylated by the microbiota or a further, rapid metabolism of fucoxanthinol into other compounds (Figure S1 of the Supporting Information). In general, there is a paucity of information on carotenoid metabolism by gut microbiota, especially for fucoxanthin. These data represent the first report on the fate of fucoxanthin in the colon phase. Because some absorption of carotenoids in the colon is likely, ${ }^{42}$ the loss of fucoxanthin credited to gut microbiota may affect its overall bioavailability.

Effect of Fucoxanthin on SCFA Production during In Vitro Colonic Fermentation. In the present study, we have investigated whether the presence of dietary fucoxanthin would affect the fermentative activity of gut microbiota from three different donors by measuring the SCFA concentration during fermentation of a basal medium with and without the addition of NLE (Figure 6). SCFA levels were different among the three donors. SCFAs produced by microbiota of donor 3 were much higher than those of donors 1 and 2. No significant changes were observed when NLE was added to microbiota from donor 1. However, SCFA production was greatly reduced when NLE

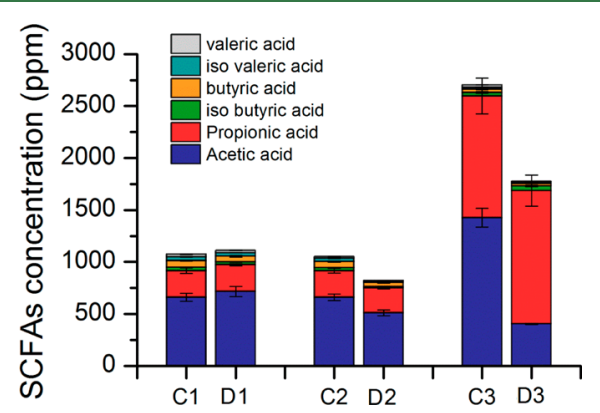

Figure 6. SCFA production after $72 \mathrm{~h}$ of in vitro colonic fermentation. All of the data were from three replications and expressed as the mean $\pm \mathrm{SD}$. C1, C2, and $\mathrm{C} 3$ represent fermentation of a basal medium without the addition of NLE to microbiota of donors 1, 2, and 3, respectively. D1, D2, and D3 represent fermentation of the basal medium with the addition of NLE to microbiota of donors 1, 2, and 3, respectively. 
was fermented by microbiota of donors 2 and 3. Specifically, acetic acid production was reduced in donors 2 and 3 but not with microbiota of donor 1. For all three treated groups, propionic acid levels were not different compared to their controls. Butyric acid production was reduced by NLE addition in donors 2 and 3 but not in donor 1 . All three SCFAs, acetic, propionic, and butyric acids, represent 90-95\% of the total SCFAs present in the colon. These SCFAs are not only crucial for gut health but might also directly affect the metabolism or the function of peripheral tissues when absorbed by the host. ${ }^{43}$ Also, SCFA production depends upon microbiota strains, and these SCFA-produced strains could be manipulated by diet. ${ }^{44}$ In this sense, the changes of SCFA production might indicate a modulation of microbiota by NLE.

In the current study, for the first time, we reported a systematic investigation of fucoxanthin deacetylation and bioaccessibility during gastrointestinal digestion and colonic fermentation. A higher recovery of fucoxanthin (in the form of fucoxanthin and fucoxanthinol) from NLE was observed in comparison to that from $\mathrm{PF}$ and FCE. Also, we provide evidence that fucoxanthin transformation into fucoxanthinol is mostly carried out by digestive lipases, most likely and primarily by pancreatic lipase, and that fucoxanthinol production follows exponential kinetics during digestion. More than $50 \%$ of fucoxanthin was lost after $24 \mathrm{~h}$ of fermentation by gut microbiota in part because of the metabolism of fucoxanthin by the gut microbiota. However, the nature and fate of fucoxanthin metabolites are still unknown and require further investigation. Similarly, bioaccessibility of fucoxanthin from common food preparations based on algae is warranted to probe the actual bioaccessibility of fucoxanthin from these sources.

\section{ASSOCIATED CONTENT}

\section{S Supporting Information}

The Supporting Information is available free of charge on the ACS Publications website at DOI: 10.1021/acs.jafc.9b02496.

Chromatogram of NLE during fermentation at $0,2,8$, 24, 48, and $72 \mathrm{~h}$ (Figure S1) (PDF)

\section{AUTHOR INFORMATION}

\section{Corresponding Authors}

*Telephone: +86-6276664. E-mail: sfchencoe@pku.edu.cn.

*Telephone: +31-317485690. E-mail: edoardo.capuano@wur. nl.

\section{ORCID}

Bingbing Guo: 0000-0003-3471-168X

Vincenzo Fogliano: 0000-0001-8786-9355

\section{Funding}

The study was supported by the China Scholarships Council.

Notes

The authors declare no competing financial interest.

\section{REFERENCES}

(1) Matsuno, T. Aquatic animal carotenoids. Fish. Sci. 2001, 67 (5), 771-783.

(2) Woo, M.-N.; Jeon, S.-M.; Shin, Y. C.; Lee, M.-K.; Kang, M. A.; Choi, M.-S. Anti-obese property of fucoxanthin is partly mediated by altering lipid-regulating enzymes and uncoupling proteins of visceral adipose tissue in mice. Mol. Nutr. Food Res. 2009, 53 (12), 16031611.
(3) Maeda, H.; Hosokawa, M.; Sashima, T.; Miyashita, K. Dietary combination of fucoxanthin and fish oil attenuates the weight gain of white adipose tissue and decreases blood glucose in obese/diabetic KK-Ay mice. J. Agric. Food Chem. 2007, 55 (19), 7701-7706.

(4) Hitoe, S.; Shimoda, H. Seaweed Fucoxanthin Supplementation Improves Obesity Parameters in Mild Obese Japanese Subjects. Funct. Foods Health Dis. 2017, 7 (4), 246-262.

(5) Knecht, L. Effects of Xanthigen Supplementation on Body Weight, Resting Energy Expenditure, and Body Composition in an Obese Population. Honors Thesis, The University of Southern Mississippi, Hattiesburg, MS, 2012; 43.

(6) Guo, B.; Liu, B.; Yang, B.; Sun, P.; Lu, X.; Liu, J.; Chen, F. Screening of diatom strains and characterization of Cyclotella cryptica as a potential fucoxanthin producer. Mar. Drugs 2016, 14 (7), 125.

(7) Mori, K.; Ooi, T.; Hiraoka, M.; Oka, N.; Hamada, H.; Tamura, M.; Kusumi, T. Fucoxanthin and its metabolites in edible brown algae cultivated in deep seawater. Mar. Drugs 2004, 2 (2), 63-72.

(8) Kanazawa, K.; Ozaki, Y.; Hashimoto, T.; Das, S. K.; Matsushita, S.; Hirano, M.; Okada, T.; Komoto, A.; Mori, N.; Nakatsuka, M. Commercial-scale preparation of biofunctional fucoxanthin from waste parts of brown sea algae Laminalia japonica. Food Sci. Technol. Res. 2008, 14 (6), 573-573.

(9) Fourest, E.; Volesky, B. Alginate properties and heavy metal biosorption by marine algae. Appl. Biochem. Biotechnol. 1997, 67 (3), 215-226.

(10) Wen, Z.; Chen, F. Heterotrophic production of eicosapentaenoid acid by the diatom Nitzschia laevis: Effects of silicate and glucose. J. Ind. Microbiol. Biotechnol. 2000, 25 (4), 218-224.

(11) Lu, X.; Sun, H.; Zhao, W.; Cheng, K.-W.; Chen, F.; Liu, B. A Hetero-Photoautotrophic Two-Stage Cultivation Process for Production of Fucoxanthin by the Marine Diatom Nitzschia laevis. Mar. Drugs 2018, 16 (7), 219.

(12) Guo, B.; Liu, B.; Wei, H.; Cheng, K. W.; Chen, F. Extract of the Microalga Nitzschia laevis Prevents High-Fat-Diet Induced Obesity in Mice by Modulating the Composition of Gut Microbiota. Mol. Nutr. Food Res. 2019, 63, 1800808.

(13) Sugawara, T.; Baskaran, V.; Tsuzuki, W.; Nagao, A. Brown algae fucoxanthin is hydrolyzed to fucoxanthinol during absorption by Caco-2 human intestinal cells and mice. J. Nutr. 2002, 132 (5), 946951.

(14) Hashimoto, T.; Ozaki, Y.; Taminato, M.; Das, S. K.; Mizuno, M.; Yoshimura, K.; Maoka, T.; Kanazawa, K. The distribution and accumulation of fucoxanthin and its metabolites after oral administration in mice. Br. J. Nutr. 2009, 102 (2), 242-248.

(15) Asai, A.; Yonekura, L.; Nagao, A. Low bioavailability of dietary epoxyxanthophylls in humans. Br. J. Nutr. 2008, 100 (2), 273-277.

(16) Hii, S.; Choong, P.; Woo, K.; Wong, C. Stability studies of fucoxanthin from Sargassum binderi. Aust. J. Basic Appl. Sci. 2010, 4 (10), 4580-4584.

(17) Salvia-Trujillo, L.; Sun, Q.; Um, B.; Park, Y.; McClements, D. In vitro and in vivo study of fucoxanthin bioavailability from nanoemulsion-based delivery systems: Impact of lipid carrier type. J. Funct. Foods 2015, 17, 293-304.

(18) Mok, I.-K.; Lee, J. K.; Kim, J. H.; Pan, C.-H.; Kim, S. M. Fucoxanthin bioavailability from fucoxanthin-fortified milk: In vivo and in vitro study. Food Chem. 2018, 258, 79-86.

(19) Bohn, T.; Desmarchelier, C.; Dragsted, L. O.; Nielsen, C. S.; Stahl, W.; Rühl, R.; Keijer, J.; Borel, P. Host-related factors explaining interindividual variability of carotenoid bioavailability and tissue concentrations in humans. Mol. Nutr. Food Res. 2017, 61 (6), 1600685 .

(20) Karlsson, F. H.; Fåk, F.; Nookaew, I.; Tremaroli, V.; Fagerberg, B.; Petranovic, D.; Bäckhed, F.; Nielsen, J. Symptomatic atherosclerosis is associated with an altered gut metagenome. Nat. Commun. 2012, 3 (1), 1245.

(21) Grolier, P.; Borel, P.; Duszka, C.; Lory, S.; Alexandre-Gouabau, M.; Azais-Braesco, V.; Nugon-Baudon, L. The bioavailability of $\alpha$-and $\beta$-carotene is affected by gut microflora in the rat. Br. J. Nutr. 1998, 80 (2), 199-204. 
(22) Djuric, Z.; Bassis, C.; Ren, J.; Chan, R.; Kato, I.; Ruffin, M. T.; Sen, A.; Turgeon, D. Predictors of Carotenoid Concentrations in Human Serum: Role of the Intestinal Microbiome. FASEB J. 2017, 31, 168.7.

(23) Verkempinck, S.; Salvia-Trujillo, L.; Moens, L.; Charleer, L.; Van Loey, A.; Hendrickx, M.; Grauwet, T. Emulsion stability during gastrointestinal conditions effects lipid digestion kinetics. Food Chem. 2018, 246, 179-191.

(24) Minekus, M.; Alminger, M.; Alvito, P.; Ballance, S.; Bohn, T.; Bourlieu, C.; Carriere, F.; Boutrou, R.; Corredig, M.; Dupont, D. A standardised static in vitro digestion method suitable for food-An international consensus. Food Funct. 2014, 5 (6), 1113-1124.

(25) Mutsokoti, L.; Panozzo, A.; Pallares Pallares, A.; Jaiswal, S.; Van Loey, A.; Grauwet, T.; Hendrickx, M. Carotenoid bioaccessibility and the relation to lipid digestion: A kinetic study. Food Chem. 2017, 232, 124-134.

(26) Bhattarai, R. R.; Dhital, S.; Gidley, M. Interactions among macronutrients in wheat flour determine their enzymic susceptibility. Food Hydrocolloids 2016, 61, 415-425.

(27) Poeker, S. A.; Geirnaert, A.; Berchtold, L.; Greppi, A.; Krych, L.; Steinert, R. E.; de Wouters, T.; Lacroix, C. Understanding the prebiotic potential of different dietary fibers using an in vitro continuous adult fermentation model (PolyFermS). Sci. Rep. 2018, 8 (1), 4318.

(28) Mosele, J. I.; Macià, A.; Romero, M.-P.; Motilva, M.-J.; Rubió, L. Application of in vitro gastrointestinal digestion and colonic fermentation models to pomegranate products (juice, pulp and peel extract) to study the stability and catabolism of phenolic compounds. J. Funct. Foods 2015, 14, 529-540.

(29) Yip, W. H.; Joe, L. S.; Mustapha, W. A. W.; Maskat, M. Y.; Said, M. Characterisation and stability of pigments extracted from Sargassum binderi obtained from Semporna, Sabah. Sains Malays. 2014, 43 (9), 1345-1354.

(30) Dressman, J. B.; Berardi, R. R.; Dermentzoglou, L. C.; Russell, T. L.; Schmaltz, S. P.; Barnett, J. L.; Jarvenpaa, K. Upper gastrointestinal (GI) $\mathrm{pH}$ in young, healthy men and women. Pharm. Res. 1990, 07 (7), 756-761.

(31) Kalantzi, L.; Goumas, K.; Kalioras, V.; Abrahamsson, B.; Dressman, J. B.; Reppas, C. Characterization of the human upper gastrointestinal contents under conditions simulating bioavailability/ bioequivalence studies. Pharm. Res. 2006, 23 (1), 165-176.

(32) Konovalov, V.; Kispert, L. Perkin Transactions 2, AM1, INDO/ $S$ and optical studies of carbocations of carotenoid molecules. Acid induced isomerization. J. Chem. Soc., Perkin Trans. 2 1999, No. 4, 901-910.

(33) Kartikaningsih, H.; Mufti, E. D.; Nurhanief, A. E. Fucoxanthin from brown seaweed Sargassum cristaefolium tea in acid pH. AIP Conf. Proc. 2017, 1844, 030009.

(34) Sugawara, T.; Baskaran, V.; Tsuzuki, W.; Nagao, A. Brown algae fucoxanthin is hydrolyzed to fucoxanthinol during absorption by Caco-2 human intestinal cells and mice. J. Nutr. 2002, 132 (5), 946951.

(35) Sugawara, T.; Matsubara, K.; Akagi, R.; Mori, M.; Hirata, T. Antiangiogenic activity of brown algae fucoxanthin and its deacetylated product, fucoxanthinol. J. Agric. Food Chem. 2006, 54 (26), 9805-9810.

(36) Zahir, M.; Fogliano, V.; Capuano, E. Food matrix and processing modulate in vitro protein digestibility in soybeans. Food Funct. 2018, 9 (12), 6326-6336.

(37) Armand, M. Lipases and lipolysis in the human digestive tract: Where do we stand? Curr. Opin. Clin. Nutr. Metab. Care 2007, 10 (2), 156-164.

(38) Mok, I.-K.; Lee, J. K.; Kim, J. H.; Pan, C.-H.; Kim, S. Fucoxanthin bioavailability from fucoxanthin-fortified milk: In vivo and in vitro study. Food Chem. 2018, 258, 79-86.

(39) Mutsokoti, L.; Panozzo, A.; Pallares Pallares, A.; Jaiswal, S.; Van Loey, A.; Grauwet, T.; Hendrickx, M. Carotenoid bioaccessibility and the relation to lipid digestion: A kinetic study. Food Chem. 2017, 232, 124-134.
(40) Salvia-Trujillo, L.; Verkempinck, S.; Sun, L.; Van Loey, A.; Grauwet, T.; Hendrickx, M. Lipid digestion, micelle formation and carotenoid bioaccessibility kinetics: Influence of emulsion droplet size. Food Chem. 2017, 229, 653-662.

(41) Hart, S. M.; Lin, X. L.; Thilakarathna, S. H.; Wright, A. Emulsion droplet crystallinity attenuates early in vitro digestive lipolysis and $\beta$-carotene bioaccessibility. Food Chem. 2018, 260, 145151.

(42) Ribeiro, H. S.; Guerrero, J. M.; Briviba, K.; Rechkemmer, G.; Schuchmann, H. P.; Schubert, H. Cellular uptake of carotenoidloaded oil-in-water emulsions in colon carcinoma cells in vitro. $J$. Agric. Food Chem. 2006, 54 (25), 9366-9369.

(43) Canfora, E. E.; Jocken, J. W.; Blaak, E. Short-chain fatty acids in control of body weight and insulin sensitivity. Nat. Rev. Endocrinol. 2015, 11 (10), 577.

(44) Reichardt, N.; Vollmer, M.; Holtrop, G.; Farquharson, F. M.; Wefers, D.; Bunzel, M.; Duncan, S. H.; Drew, J. E.; Williams, L. M.; Milligan, G.; Preston, T.; Morrison, D.; Flint, H. J.; Louis, P. Specific substrate-driven changes in human faecal microbiota composition contrast with functional redundancy in short-chain fatty acid production. ISME J. 2018, 12 (2), 610-622. 